\title{
ON A METHOD OF HOLOMORPHIC FUNCTIONS TO OBTAIN SHARP REGULARIZATION RATES OF WEAK SOLUTIONS OF NAVIER-STOKES EQUATIONS*
}

\author{
EUGENE TSYGANOV ${ }^{\dagger}$
}

\begin{abstract}
We show that $L^{2}$ energy estimates combined with Cauchy integral formula for holomorphic functions can provide bounds for higher-order derivatives of smooth solutions of NavierStokes equations. We then extend this principle to weak solutions to improve regularization rates obtained by standard energy methods.
\end{abstract}

Key words. Compressible Navier-Stokes equations, weak solutions, time analyticity, holomorphic functions.

AMS subject classifications. 35B35, 35B40, 76N10

1. Introduction. We show that there is a weak solution of the Navier-Stokes equations

$$
\begin{aligned}
& v_{t}-u_{x}=0, \\
& u_{t}+p_{x}=\left(\varepsilon \frac{u_{x}}{v}\right)_{x}, \\
& \left(e+\frac{1}{2} u^{2}\right)_{t}+(u p)_{x}=\left(\kappa \frac{e_{x}}{v}+\varepsilon \frac{u u_{x}}{v}\right)_{x}
\end{aligned}
$$

with optimal regularization rates. Here $t \geq 0$ is time, $x \in \mathbb{R}$ is the spatial coordinate, $v$ is the specific volume, $e$ is the internal energy, positive constants $\varepsilon, \kappa$ are the viscosity coefficient and the heat conductivity. We assume that the fluid is ideal, so that $P(v, e)=(\gamma-1) \frac{e}{v}$, where $\gamma>1$ is the adiabatic constant.

The questions addressed in the paper are motivated by the fact that regularization rates play a critical role in proving uniqueness and continuous dependence on initial data of weak solutions of Navier-Stokes equations. Although the question of uniqueness was resolved in Hoff \& Tsyganov [2], the issue of small-time behavior of weak solutions remained. We would also like to point out that there exist other methods of proving uniqueness without knowing regularization rates (see Zlotnik \& Amosov [6] and Jiang \& Zlotnik [4]).

It is known that solutions of the heat equation $u_{t}=u_{x x}, u(\cdot, 0)=u_{0}(\cdot) \in L^{2}(\mathbb{R})$ become smooth for $t>0$ and satisfy the inequalities

$$
t \int_{\mathbb{R}} u_{x}^{2}(x, t) d x+t^{2} \int_{\mathbb{R}} u_{t}^{2}(x, t) d x \leq \int_{\mathbb{R}} u_{0}^{2}(x) d x, 0 \leq t \leq 1,
$$

and the regularization rates $1 / t$ for $\left\|u_{x}(\cdot, t)\right\|_{L^{2}(\mathbb{R})}^{2}$ and $1 / t^{2}$ for $\left\|u_{t}(\cdot, t)\right\|_{L^{2}(\mathbb{R})}^{2}$ are considered to be sharp. A similar parabolic smoothing mechanism is also present in Navier-Stokes equations. As can be seen from the existence result of Chen, Hoff, Trivisa [1], $u_{x}, e_{x}, u_{t}, e_{t}$ are in $L^{2}(\mathbb{R})$ for $t>0$, but their rates of regularization are not optimal. The present paper therefore is the first result presenting an efficient method of obtaining sharp estimates for hyperbolic-parabolic differential equations with high-order nonlinear terms.

\footnotetext{
* Received December 25, 2007; accepted for publication June 19, 2008.

†Bashkir State University, Russia (entsyganov@yahoo.com).
} 
Time analyticity of weak solutions of compressible Navier-Stokes equations, which was established in Tsyganov [5] and Hoff \& Tsyganov [3], finds several important applications. In the current paper we present a method of obtaining higher-order estimates from $L^{2}$ bounds for complex values of $t$ by means of Cauchy integral formula for holomorphic functions. This new approach allows us to overcome difficulties arising in the standard analysis.

We now give a precise formulation of our results. We say that $(v, u, e)$ is a weak solution of $(1.1)-(1.3)$ on $[0, T]$ if for every test function $\varphi, \psi \in \mathscr{D}(\mathbb{R} \times(-\infty, \infty))$ and all times $0 \leq t_{1}<t_{2} \leq T$,

$$
\begin{gathered}
\left.\int_{\mathbb{R}} v \varphi d x\right|_{t_{1}} ^{t_{2}}-\int_{t_{1}}^{t_{2}} \int_{\mathbb{R}}\left[v \varphi_{t}-u \varphi_{x}\right] d x d t=0 \\
\left.\int_{\mathbb{R}} u \psi d x\right|_{t_{1}} ^{t_{2}}-\int_{t_{1}}^{t_{2}} \int_{\mathbb{R}}\left[u \psi_{t}+p \psi_{x}\right] d x d t=-\int_{t_{1}}^{t_{2}} \int_{\mathbb{R}} \varepsilon \frac{u_{x}}{v} \psi_{x} d x d t \\
\left.\int_{\mathbb{R}}\left(e+\frac{1}{2} u^{2}\right) \chi d x\right|_{t_{1}} ^{t_{2}}-\int_{t_{1}}^{t_{2}} \int_{\mathbb{R}}\left(e+\frac{1}{2} u^{2}\right) \chi_{t} d x d t-\int_{t_{1}}^{t_{2}} \int_{\mathbb{R}} u p \chi_{x} d x d t \\
=-\int_{t_{1}}^{t_{2}} \int_{\mathbb{R}}\left(\kappa \frac{e_{x}}{v}+\varepsilon \frac{u u_{x}}{v}\right) \chi_{x} d x d t
\end{gathered}
$$

The following theorem contains our main result:

TheOREM 1.1. Let $\tilde{v}, \tilde{e}, \underline{e}, M, \mu$ be positive constants such that $M^{-1}-\mu, \underline{e}-\mu>0$. Then given initial data $\left(v_{0}, u_{0}, e_{0}\right)$ for which

$$
\begin{gathered}
C_{0} \equiv \int_{-\infty}^{\infty}\left(v_{0}(x)-\tilde{v}\right)^{2} d x+\int_{-\infty}^{\infty} u_{0}^{2}(x) d x+\int_{-\infty}^{\infty}\left(e_{0}(x)-\tilde{e}\right)^{2} d x \\
+\int_{-\infty}^{\infty} u_{0}^{4}(x) d x<\infty
\end{gathered}
$$

and

$$
v_{0} \in\left[M^{-1}, M\right] \text { a.e. }, e_{0} \in[\underline{e}, \infty) \text { a.e., }
$$

there is a positive $T$ depending on $\tilde{v}, \tilde{e}, \underline{e}, \mu, M, C_{0}$, a positive constant $C$ depending on $\tilde{v}, \tilde{e}, \underline{e}, \mu, M$, and a local weak solution $(v, u, e)$ of $(1.1)-(1.3)$ on $[0, T]$, satisfying

$$
\begin{gathered}
v \in\left[M^{-1}-\mu, M+\mu\right] \text { a.e. }, e \in[\underline{e}-\mu, \infty), \\
\sup _{t \in[0, T]}\left[\int_{\mathbb{R}}\left[(v(x, t)-\tilde{v})^{2}+u^{2}(x, t) d x+(e(x, t)-\tilde{e})^{2}+u^{4}(x, t)\right] d x\right] \\
+\int_{0}^{T} \int_{\mathbb{R}}\left[u_{x}^{2}(x, s)+e_{x}^{2}(x, s)+u^{2}(x, s) u_{x}^{2}(x, s)\right] d x d s \leq C C_{0}, \\
\sup _{t \in[0, T]}\left[t \int_{\mathbb{R}}\left[u_{x}^{2}(x, t)+e_{x}^{2}(x, t)+u^{2}(x, t) u_{x}^{2}(x, t)\right] d x\right. \\
\left.+t^{2} \int_{\mathbb{R}}\left[u_{t}^{2}(x, t)+e_{t}^{2}(x, t)+u^{2}(x, t) u_{t}^{2}(x, t)\right] d x\right] \leq C C_{0} .
\end{gathered}
$$


We now give a brief overview of the analysis presented in the paper. The weak solution $(v, u, e)$ will be obtained as a limit of smooth solutions of (1.1)-(1.3) for mollified initial data. The main idea lies in a sequence of a priori bounds, which are connected as follows:

I. We construct an open set $\mathcal{D} \subset \mathbb{C}$ with specific convexity properties, then we apply standard existence results to find a complex smooth solution of (1.1) - (1.3) for $t \in \mathcal{D}$;

II. Assuming that $v$ satisfies pointwise estimates (1.6) in $\mathcal{D}$, we show via compexification of equations $(1.1)-(1.3)$ that $\|v(\cdot, t)-\tilde{v}\|_{L^{2}(\mathbb{R})}$, $\|u(\cdot, t)\|_{L^{2}(\mathbb{R})},\|e(\cdot, t)-\tilde{e}\|_{L^{2}(\mathbb{R})},\left\|u^{2}(\cdot, t)\right\|_{L^{2}(\mathbb{R})}$, as well as line integrals from 0 to $t$ of $\left\|u_{x}(\cdot, s)\right\|_{L^{2}(\mathbb{R})}^{2},\left\|e_{x}(\cdot, s)\right\|_{L^{2}(\mathbb{R})}^{2},\left\|u(\cdot, s) u_{x}(\cdot, s)\right\|_{L^{2}(\mathbb{R})}^{2}$ are bounded by $C C_{0}$ for any $t \in \mathcal{D}$;

III. We assume that the estimates in II hold and exploit convexity of $\mathcal{D}$ to obtain bounds for $\left\|u_{t}(\cdot, t)\right\|_{L^{2}(\mathbb{R})},\left\|e_{t}(\cdot, t)\right\|_{L^{2}(\mathbb{R})},\left\|u(\cdot, t) u_{t}(\cdot, t)\right\|_{L^{2}(\mathbb{R})}$ and $\left\|u_{x}(\cdot, t)\right\|_{L^{2}(\mathbb{R})},\left\|e_{x}(\cdot, t)\right\|_{L^{2}(\mathbb{R})},\left\|u(\cdot, t) u_{x}(\cdot, t)\right\|_{L^{2}(\mathbb{R})}$ directly from Cauchy integral formula for holomorphic functions;

IV. We derive pointwise bounds for $v$ from the estimates in III and the inequality $\left|v(x, t)-v_{0}(x)\right| \leq C \int_{0}^{t}\left\|u_{x}(\cdot, s)\right\|_{L^{\infty}(\mathbb{R})}$.

2. Construction of a Special Domain and $L^{2}$ Bounds. In this section we construct a special domain in $\mathbb{C}$ and obtain a priori $L^{2}$ estimates for time values from this set.

We begin with construction of a specific domain in $\mathbb{C}$ whose properties will play a key role in the analysis.

LEMMA 2.1. For any $n>0$ there are positive constants $C, \bar{\theta}, b$, and a convex open set $\mathcal{D}$ in $\mathbb{C}$, all as small as necessary, such that

1) $\mathcal{D} \subset\{z:|\arg (z)|<\bar{\theta}, \operatorname{Re}(z)>0,|z| \leq b\}$

2) $\mathcal{D}$ is symmetric with respect to $\{z: \operatorname{Im}(z)=0\}$;

3) $0 \in \partial \mathcal{D}$;

4) for any $z \in \mathcal{D}$ there are distinct $z_{1}, z_{2} \in \mathcal{D}$ such that

$$
\begin{aligned}
& \left|z_{1}\right|=\left|z_{1}-z\right|=\left|z_{2}\right|=\left|z_{2}-z\right|, \\
& |z| \leq 2^{n+1} \sin ^{n}\left(\angle z_{1} 0 z\right),
\end{aligned}
$$

where $\angle z_{1} 0 z$ is the angle between two lines $0 z_{1}$ and $0 z$.

Proof. We fix an arbitrary positive $\lambda<1$ and locate a point $A_{1}=\lambda$ on the $\operatorname{Re}(z)$ axis. Let $A_{2}$ be the point with the following properties:

$$
\left|A_{2}-A_{1}\right|=\left|A_{2}\right|, \quad \sin ^{n}\left(\angle A_{2} 0 A_{1}\right)=\lambda .
$$

Assume now that we have constructed $k-1$ vertices $A_{1}, \ldots, A_{k-1}$. Then the $k-t h$ vertex $A_{k}$ satisfies

$$
\left|A_{k}-A_{k-1}\right|=\left|A_{k}\right|, \quad \sin ^{n}\left(\angle A_{k} 0 A_{k-1}\right)=\left|A_{k-1}\right| .
$$


In addition, let $A_{k}^{*}$ be the reflection of $A_{k}$ with respect to the $\operatorname{Re}(z)$ axis. We then define $\mathcal{D}$ as an open set bounded by the union of line segments $A_{k} A_{k-1}, A_{k}^{*} A_{k-1}^{*}, k=$ $1,2, \ldots$, and $z=0$.

First we show that $\sup _{k}\left(\operatorname{Arg}\left(A_{k}\right)\right) \rightarrow 0$ when $\lambda \rightarrow 0$. We have for $\lambda \ll 1$ that

$$
\begin{aligned}
\operatorname{Arg}\left(A_{k}\right) & =\sum_{m=2}^{k}\left(\angle A_{m} 0 A_{m-1}\right)=\sum_{m=2}^{k} \arcsin \left(\left|A_{m-1}\right|^{1 / n}\right) \\
& <2 \sum_{m=1}^{k}\left|A_{m}\right|^{1 / n}<2 \sum_{m=0}^{\infty}\left(\lambda\left(\frac{2}{3}\right)^{m}\right)^{1 / n}=\lambda^{1 / n} \frac{2}{1-\left(\frac{2}{3}\right)^{1 / n}}
\end{aligned}
$$

so that $\mathcal{D}$ will satisfy condition 1 ) if $\lambda$ is small enough. Next we take an arbitrary $z \in \mathcal{D}$. Due to symmetry of $\mathcal{D}$ we can assume that $\operatorname{Im}(z)>0$. Then $z$ belongs to a certain triangle $\triangle O A_{k-1} A_{k}$, where $O$ is the origin. The absolute value $|z|$ is less than $\left|A_{k-1}\right|$ and there is $z_{1} \in \triangle O A_{k} A_{k+1}$ such that $\left|z_{1}\right|=\left|z_{1}-z\right|$ and $\angle z_{1} 0 z>$ $\frac{1}{2} \angle A_{k+1} 0 A_{k}$. Therefore, $|z|<2^{n+1} \sin ^{n}\left(\angle z_{1} 0 z\right)$.

LEMMA 2.2. There exists a positive $\tau<1$ depending on the same quantities as $C$ in the statement of Theorem 1.1 such that if $\mathcal{D}$ is an open set with properties 1)-4) of Lemma 1.1 satisfying

$$
\mathcal{D} \subset\{z:|\arg (z)| \leq \tau,|z| \leq \tau\}
$$

and if $(v, u, e)$ is any smooth solution of (1.1)-(1.3) satisfying the following conditions:

$$
\begin{aligned}
& v(x, t), u(x, t), e(x, t) \text { are holomorphic in } \mathcal{D} \text { with respect to } t \\
& \text { for any fixed } x \in \mathbb{R} ; \\
& \text { for any } \theta,|\theta|=1,|\arg (\theta)| \leq \tau, \text { smooth functions } \\
& \quad(v(x, \theta s), u(x, \theta s), e(x, \theta s)) \text { of }(x, s) \text { solve }
\end{aligned}
$$

$$
\begin{aligned}
& v_{s}-\bar{\theta} u_{x}=0 \\
& u_{s}+\bar{\theta} p_{x}=\bar{\theta}\left(\varepsilon \frac{u_{x}}{v}\right)_{x}, \\
& \left(e+\frac{1}{2} u^{2}\right)_{s}+\bar{\theta}(u p)_{x}=\bar{\theta}\left(\kappa \frac{e_{x}}{v}+\varepsilon \frac{u u_{x}}{v}\right)_{x}
\end{aligned}
$$

at any point $(x, s)$ for which $\theta s \in \mathcal{D}$;

$$
M^{-1}-\mu \leq \operatorname{Re}(v(x, t)) \leq M+\mu, \quad|\operatorname{Im}(v(x, t))| \leq \mu, \quad t \in \mathcal{D},
$$

then

$$
\begin{gathered}
\int\left[|v(x, \theta s)-\tilde{v}|^{2}+|u(x, \theta s)|^{2}+|e(x, \theta s)-\tilde{e}|^{2}+|u(x, \theta s)|^{4}\right] d x \\
+\int_{0}^{T} \int_{\mathbb{R}}\left[\left|u_{x}(x, \theta s)\right|^{2}+\left|e_{x}(x, \theta s)\right|^{2}+\left|u(x, \theta s) u_{x}(x, \theta s)\right|^{2}\right] d x d s \leq C C_{0},
\end{gathered}
$$

as long as $\theta s \in \mathcal{D}$. Here $C$ is a positive constant as described in Theorem 1.1. 
Proof. We multiply (2.1) by $\bar{v}-\tilde{v},(2.2)$ by $\bar{u},(2.3)$ by $\bar{e}-\tilde{e}$, add the resulting equations and integrate. Then we add the equation to its conjugate to arrive at

$$
\begin{gathered}
\left.\int\left[|v(x, s)-\tilde{v}|^{2}+|u(x, s)|^{2}+|e(x, s)-\tilde{e}|^{2}\right] d x\right|_{s=0} ^{s=t} \\
+\int_{0}^{t} \int\left[\varepsilon\left(\frac{\bar{\theta}}{v}+\frac{\theta}{\bar{v}}\right)\left|u_{x}\right|^{2}+\kappa\left(\frac{\bar{\theta}}{v}+\frac{\theta}{\bar{v}}\right)\left|e_{x}\right|^{2}\right] d x d s \\
=-\int_{0}^{t} \int\left[u u_{t}(\bar{e}-\tilde{e})+\bar{u} \bar{u}_{t}(e-\tilde{e})+\varepsilon \theta \frac{u u_{x} \bar{e}_{x}}{v}+\varepsilon \bar{\theta} \frac{\bar{u} \bar{u}_{x} e_{x}}{\bar{v}}\right] d x d s \\
-\int_{0}^{t} \int\left[\bar{\theta} u_{x}(\bar{v}-\tilde{v})+\theta \bar{u}_{x}(v-\tilde{v})-\bar{\theta}(\bar{p}-\tilde{p}) u_{x}-\theta(p-\tilde{p}) \bar{u}_{x}\right] d x d s \\
+\int_{0}^{t} \int\left[\bar{\theta} u p \bar{e}_{x}+\theta \bar{u} \bar{p} e_{x}\right] d x d s .
\end{gathered}
$$

We then multiply $(2.2)$ by $u \bar{u}^{2}$, integrate, and add to its conjugate to obtain

$$
\begin{aligned}
& \left.\int|u(x, s)|^{4} d x\right|_{s=0} ^{s=t}+\int_{0}^{t} \int 2 \varepsilon\left(\frac{\bar{\theta}}{v}+\frac{\theta}{\bar{v}}\right)|u|^{2}\left|u_{x}\right|^{2} d x d s \\
& =\int_{0}^{t} \int\left[\varepsilon \bar{\theta} \frac{u^{2} \bar{u}_{x}^{2}}{v}+\varepsilon \theta \frac{\bar{u}^{2} u_{x}^{2}}{\bar{v}}\right] d x d s \\
& +\int_{0}^{t} \int\left[\bar{\theta}(p-\tilde{p}) u_{x} \bar{u}^{2}+2 \bar{\theta}(p-\tilde{p}) u \bar{u} \bar{u}_{x}\right] d x d s \\
& +\int_{0}^{t} \int\left[\theta(\bar{p}-\tilde{p}) \bar{u}_{x} u^{2}+2 \theta(\bar{p}-\tilde{p}) \bar{u} u u_{x}\right] d x d s .
\end{aligned}
$$

We also multiply (2.3) by $\frac{1}{2} \bar{u}^{2}$ and integrate. Then we add the equation to its conjugate to arrive at

$$
\begin{aligned}
\left.\frac{1}{4} \int|u(x, s)|^{4} d x\right|_{0} ^{t}+\frac{1}{2} \int_{0}^{t} \int\left[e_{t} \bar{u}^{2}+\bar{e}_{t} u^{2}\right] d x d s \\
=-\int_{0}^{t} \int\left[\kappa \bar{\theta} \frac{e_{x} \bar{u} \bar{u}_{x}}{v}+\kappa \theta \frac{\bar{e}_{x} u u_{x}}{\bar{v}}+\varepsilon\left(\frac{\bar{\theta}}{v}+\frac{\theta}{\bar{v}}\right)|u|^{2}\left|u_{x}\right|^{2}\right] d x d s \\
\quad+\int_{0}^{t} \int\left[\bar{\theta} u p \bar{u} \bar{u}_{x}+\theta \bar{u} \bar{p} u u_{x}\right] d x d s .
\end{aligned}
$$

For a smooth solution $(v, u, e)$ of $(2.1)-(2.3)$ we define

$$
\begin{aligned}
A(t)= & \sup _{0 \leq s \leq t} \int\left[|v(x, \theta s)-\tilde{v}|^{2}+|u(x, \theta s)|^{2}+|e(x, \theta s)-\tilde{e}|^{2}+|u(x, \theta s)|^{4}\right] d x \\
& +\int_{0}^{t} \int\left[\left|u_{x}(x, \theta s)\right|^{2}+\left|e_{x}(x, \theta s)\right|^{2}+|u(x, \theta s)|^{2}\left|u_{x}(x, \theta s)\right|^{2}\right] d x d s .
\end{aligned}
$$

We now add (2.6), (2.7), and (2.8) multiplied by a suitable constant, apply CauchySchwarz inequality in an elementary way to obtain

$$
A(t) \leq C C_{0}+C \int_{0}^{t} \int|u|^{2}|e-\tilde{e}|^{2} d x d s .
$$


The double integral on the right-hand side of (2.10) can be bounded by

$$
\begin{aligned}
& \sup _{0 \leq s \leq t}\left(\int|u(x, \theta s)|^{4} d x\right)^{1 / 2}\left(\int_{0}^{t} \int|e(x, \theta s)-\tilde{e}|^{4} d x d s\right)^{1 / 2} \\
& \quad \leq C A^{1 / 2} \int_{0}^{t}\left(\int|e-\tilde{e}|^{2} d x\right)\left(\int\left|e_{x}\right|^{2} d x\right)^{1 / 2} d s \leq C C_{0} t^{1 / 2} A(t) .
\end{aligned}
$$

This together with (2.10) proves the lemma.

3. Higher-Order Estimates. In this section we obtain a priori higher-order estimates for smooth solutions utilizing only $L^{2}$ estimates and properties of $\mathcal{D}$.

LEMma 3.1. Let $\mathcal{D}$ be an open set and $(v, u, e)$ a smooth solution of (1.1)-(1.3) as described in the statement of Lemma 2.2. Then there is a positive constant $C$ depending on the same quantities as $C$ in the conclusion of Theorem 1.1 such that

$$
\left(\int\left[\left|u_{t}(x, \theta s)\right|^{2}+\left|e_{t}(x, \theta s)\right|^{2}\right] d x\right)^{1 / 2} \leq C C_{0}\left(\frac{1}{s}+\frac{1}{T-s}\right) T^{-1 / 2 n}
$$

where $\theta$ and $T=T(\theta)$ are such that $|\theta|=1$ and $T=\sup \{s: \theta s \in \mathcal{D}\}>0$.

Proof. First we fix $\theta$. Property 4) of $\mathcal{D}$ will guarantee that there exists $\beta>0$ such that $\operatorname{CSin}^{n}(\beta)>T$ and if $s \in(0, T)$, then either the disk $B(\theta s, s \beta)$ centered at $\theta s$ with radius $s \beta$ or the disk $B(\theta s,(T-s) \beta)$ centered at $\theta s$ with radius $(T-s) \beta$ lies in $\mathcal{D}$. Then from Cauchy integral formula

$$
u_{t}(x, \theta s)=\frac{1}{2 \pi i} \int_{\partial B(\theta s, \min (s \beta,(T-s) \beta))} \frac{u(x, \xi)}{(\theta s-\xi)^{2}} d \xi
$$

we can derive that

$$
\begin{aligned}
\int\left|u_{t}(x, \theta s)\right|^{2} d x & \leq C\left(\frac{1}{(s \beta)^{2}}+\frac{1}{((T-s) \beta)^{2}}\right) \sup _{t \in \mathcal{D}} \int|u(x, t)|^{2} d x \\
& \leq C C_{0}\left(\frac{1}{s^{2} \operatorname{Sin}^{2}(\beta)}+\frac{1}{(T-s)^{2} \operatorname{Sin}^{2} \beta}\right) \\
& \leq C C_{0}\left(\frac{1}{s^{2}}+\frac{1}{(T-s)^{2}}\right) T^{-1 / n}
\end{aligned}
$$

We can apply a similar argument to estimate $\int\left|e_{t}(x, \theta s)\right|^{2} d x$ and thus prove (3.1).

Lemma 3.2. Let $\mathcal{D}$ and $(v, u, e)$ be as in the statement in Lemma 2.2. Then there is a positive constant $C$ depending on the same quantities as $C$ in the conclusion of Theorem 1.1 such that

$$
\begin{gathered}
\int\left[\left|u_{x}(x, \theta s)\right|^{2}+\left|e_{x}(x, \theta s)\right|^{2}+|u(x, \theta s)|^{2}\left|u_{x}(x, \theta s)\right|^{2}\right] d x \\
\leq C C_{0}\left(\frac{1}{s}+\frac{1}{T-s}\right) T^{-1 / n} .
\end{gathered}
$$

Proof. We fix $\theta$ and find $\beta$ as in the proof of Lemma 3.1. Then from Cauchy integral formula we have that

$$
u_{x}^{2}(x, \theta s) d x=\frac{1}{2 \pi i} \int_{\partial B(\theta s, \min (s \beta,(T-s) \beta))} \frac{\int_{0}^{\xi} u_{x}^{2}(x, \zeta) d \zeta}{(\theta s-\xi)^{2}} d \xi,
$$


where $\int_{0}^{\xi} u_{x}^{2}(x, \zeta) d \zeta$ is a line integral from 0 to $\xi$. It immediately follows from (3.4) and properties of $\beta$ that

$$
\begin{aligned}
\int\left|u_{x}(x, \theta s)\right|^{2} d x & \leq C \sup _{t}\left[\int_{0}^{t} \int\left|u_{x}(x, \zeta)\right|^{2} d x d \zeta\right]\left(\frac{1}{s}+\frac{1}{T-s}\right) T^{-1 / n} \\
& \leq C C_{0}\left(\frac{1}{s}+\frac{1}{T-s}\right) T^{-1 / n}
\end{aligned}
$$

The same argument can be used to prove the estimates for $\int\left|e_{x}(x, \theta s)\right|^{2} d x$ and $\int|u(x, \theta s)|^{2}\left|u_{x}(x, \theta s)\right|^{2} d x$.

LEMma 3.3. Let $\mathcal{D}$ and $(v, u, e)$ be as in the statement of Lemma 2.2 and $\tau$ as in the conclusion of Lemma 2.2. Then

$$
M^{-1}-\mu / 2 \leq \operatorname{Re}(v(x, t)) \leq M+\mu / 2, \quad|\operatorname{Im}(v(x, t))| \leq \mu / 2, \quad t \in \mathcal{D}
$$

if $\tau$ is small enough.

Proof. We divide (2.1) by $v$ and integrate with respect to $s$ to obtain

$$
\begin{aligned}
\mid \ln \left(v(x, \theta s)-\ln \left(v_{0}(x)\right)|=| \bar{\theta} \int_{0}^{t} \frac{u_{x}}{v}(x, \theta s) d s \mid\right. \\
\leq C \int_{0}^{t}\left\|\left(\frac{u_{x}}{v}-p+\tilde{p}\right)(\cdot, \theta s)\right\|_{L^{\infty}} d s+C \int_{0}^{t}\|(p-\tilde{p})(\cdot, \theta s)\|_{L^{\infty}} d s \\
\leq C \int_{0}^{t}\left(\int\left|\frac{u_{x}}{v}-p+\tilde{p}\right|^{2} d x\right)^{1 / 4}\left(\int\left|u_{t}\right|^{2} d x\right)^{1 / 4} d s \\
\quad+C C_{0}^{1 / 2} T^{-1 / 4 n} \int_{0}^{t}\left(\frac{1}{s^{1 / 4}}+\frac{1}{(T-s)^{1 / 4}}\right) d s \\
\leq C C_{0}^{1 / 2} T^{-1 / 2 n} \int_{0}^{t}\left(\frac{1}{s^{3 / 4}}+\frac{1}{(T-s)^{3 / 4}}\right) d s+C C_{0}^{1 / 2} T^{3 / 4-1 / 4 n} \\
\leq C C_{0}^{1 / 2} T^{1 / 2-1 / 2 n}
\end{aligned}
$$

so that the right-hand side of the inequality is as small as nesessary if we choose $\tau$ small enough.

4. Existence. In this section we apply a priori bounds of Sections 2,3 to obtain the existence of weak solution $(v, u, e)$.

Let $\left(v_{0}, u_{0}, e_{0}\right)$ be initial data as described in Theorem 1.1. We define $v_{0}^{\delta}=$ $j_{\delta} * v_{0}, u_{0}^{\delta}=j_{\delta} * u_{0}, e_{0}^{\delta}=j_{\delta} * e_{0}$, where $j_{\delta}$ is the standard mollifier. In the following lemma we state local existence for smooth initial data.

Lemma 4.1. There exists $\tilde{\theta} \in \mathbb{C},|\tilde{\theta}|=1, \operatorname{Re}(\tilde{\theta})>0$ depending on $\tilde{v}, \tilde{e}, \underline{e}, \mu, M$, a positive $T$ depending on $\tilde{v}, \tilde{e}, \underline{e}, M, \mu, \delta, C_{0}$, and a positive constant $C$ depending on $\tilde{v}, \tilde{e}, \underline{e}, \mu, M$ such that there is a smooth solution $\left(v^{\delta}, u^{\delta}, e^{\delta}\right)$ satisfying the following 
regularity conditions:

$$
\begin{aligned}
& v^{\delta}(x, \cdot), u^{\delta}(x, \cdot), e^{\delta}(x, \cdot) \text { are holomorphic in } \\
& \mathcal{P}=\{z:|z| \leq T,|\arg (z)| \leq \tilde{\theta}\} \text { for any } x \in \mathbb{R} ; \\
& \left(v^{\delta}(x, \theta s), u^{\delta}(x, \theta s), e^{\delta}(x, \theta s)\right) \text { as functions of }(x, s) \\
& \text { satisfy }(2.1)-(2.3) \text { for any } \theta \in \mathbb{C},|\theta|=1,|\arg (\theta)| \leq \arg (\tilde{\theta}) \\
& M^{-1}-\mu \leq|v(x, t)| \leq M+\mu,|\operatorname{Im}(v(x, t))| \leq \mu \\
& \quad \text { for any } t \in \mathbb{C},|t| \leq T,|\arg (t)| \leq \arg (\tilde{\theta}) \\
& \left\|v^{\delta}(\cdot, t)-\tilde{v}\right\|_{H^{3}(\mathbb{R})}+\left\|u^{\delta}(\cdot, t)\right\|_{H^{4}(\mathbb{R})}+\left\|e^{\delta}(\cdot, t)-\tilde{e}\right\|_{H^{4}(\mathbb{R})} \\
& \leq C\left(\left\|v_{0}^{\delta}-\tilde{v}\right\|_{H^{3}(\mathbb{R})}+\left\|u_{0}^{\delta}\right\|_{H^{4}(\mathbb{R})}+\left\|e_{0}^{\delta}-\tilde{e}\right\|_{H^{4}(\mathbb{R})}\right) \\
& \text { for any } t \in \mathbb{C},|t| \leq T,|\arg (t)| \leq \arg (\tilde{\theta}) .
\end{aligned}
$$

Proof. The proof is conceptually very close to the one that can be found in Hoff \& Tsyganov [3] for local smooth solution of multidimensional Navier-Stokes equations. We omit the details, pointing out that $(v, u, e)$ is obtained as a limit of solutions of semi-discrete difference schemes that approximate equations (2.1)-(2.3) when the mesh parameter goes to zero.

Lemma 4.2. Let positive constants $\tilde{v}, \tilde{e}, \underline{e}, M, C_{0}$ be given. Then there exists an open set $\mathcal{D}$ as described in Lemma 2.1 depending on these parameters and a smooth solution $\left(v^{\delta}, u^{\delta}, e^{\delta}\right)$ with initial data $\left(v_{0}^{\delta}, u_{0}^{\delta}, e_{0}^{\delta}\right)$ satisfying (1.4) as described in the statement of Lemma 2.2 .

Proof. First we apply Lemma 4.1 to find $\tilde{\theta}, T$, and a smooth solution $\left(v^{\delta}, u^{\delta}, e^{\delta}\right)$ in $\mathbb{R} \times \mathcal{P}$. Then we can find an open set $\mathcal{D} \subset \mathcal{P}$ with properties 1)-4) as described in Lemma 2.1 and prove $(2.5),(3.1),(3.3),(3.5)$ for $t \in \mathcal{D}$. We can reapply local existence theorem for smooth initial data to show that $\left(v^{\delta}, u^{\delta}, e^{\delta}\right)$ can be extended into a bigger domain $\alpha \mathcal{D}, \alpha>1$. It can also be shown by standard energy estimates that (4.4) holds as long as (2.4), (2.5), and (4.3) are satisfied, so that we can repeat the entire argument again.

In the following lemma we extract a subsequence $\delta_{k} \rightarrow 0$ for which $\left(v^{\delta_{k}}, u^{\delta_{k}}, e^{\delta_{k}}\right) \rightarrow(v, u, e)$ in an appropriate sense.

Lemma 4.3. There is a sequence $\delta=\delta_{k} \rightarrow 0$ and limiting $(v, u, e)$ for which

$$
\begin{aligned}
& u^{\delta}(\cdot, t)-u(\cdot, t), e^{\delta}(\cdot, t)-e(\cdot, t) \rightarrow 0 \text { in } L_{\text {loc }}^{2}(\mathbb{R}), t \in \mathcal{D} ; \\
& u^{\delta}-u, e^{\delta}-e \rightarrow 0 \text { uniformly on compact subsets of } \mathbb{R} \times \mathcal{D} ; \\
& u_{x}^{\delta}(\cdot, t)-u_{x}(\cdot, t), e_{x}^{\delta}(\cdot, t)-e^{\delta}(\cdot, t) \rightarrow 0 \text { in } L^{2}(\mathbb{R}), t \in \mathcal{D} ; \\
& v^{\delta}-v \rightarrow 0 \text { in } L_{\text {loc }}^{2}(\mathbb{R}),
\end{aligned}
$$

where $\mathcal{D}$ is as in the statement of Lemma 4.2 .

Proof. The compactness results (4.5), (4.6), (4.7) follow immediately from the bounds $(2.5),(3.1),(3.3),(3.5)$. In order to prove (4.8) we need to introduce the effective viscous flux $F$ by

$$
F=\varepsilon \frac{u_{x}}{v}-p(v, e)+p(\tilde{v}, \tilde{e}) .
$$


From the equalities

$$
F_{x}^{\delta}=\theta u_{t}^{\delta}, \quad F_{t}^{\delta}=\varepsilon \frac{u_{t x}^{\delta}}{v^{\delta}}-\varepsilon \frac{u_{x}^{\delta^{2}}}{v^{\delta^{2}}}-p_{v}\left(v^{\delta}, e^{\delta}\right) u_{x}^{\delta}-p_{e}\left(v^{\delta}, u^{\delta}\right) e_{t}^{\delta}
$$

and the estimates $(2.5),(3.1),(3.3),(3.5)$ we can conclude that $F$ is Hölder continuous in $x$ and $t$ so that there is a sequence $\delta=\delta_{k} \longrightarrow 0$ and a limiting $F$ such that

$$
F^{\delta} \longrightarrow F \text { uniformly on compact subsets of } \mathbb{R} \times \mathcal{D} \text {. }
$$

If we rewrite (2.1) as

$$
(\ln (v))_{t}=\bar{\theta} \frac{1}{\varepsilon} F-\bar{\theta} \frac{1}{\varepsilon}(p-\tilde{p})
$$

then

$$
\begin{aligned}
\int_{-L}^{L} \mid v^{\delta_{1}}( & x, \theta t)-\left.v^{\delta_{2}}(x, \theta t)\right|^{2} d x \leq C \int_{L}^{L}\left|v_{0}^{\delta_{1}}(x)-v_{0}^{\delta_{2}}(x)\right|^{2} d x \\
& +C \int_{0}^{t} \int_{-L}^{L}\left|v^{\delta_{1}}(x, \theta s)-v^{\delta_{2}}(x, \theta s)\right|^{2} d x d s \\
& +C \int_{0}^{t} \int_{-L}^{L}\left|e^{\delta_{1}}(x, \theta s)-e^{\delta_{2}}(x, \theta s)\right|^{2} d x d s \\
& +C \int_{0}^{t} \int_{-L}^{L}\left|F^{\delta_{1}}(x, \theta s)-F^{\delta_{2}}(x, \theta s)\right|^{2} d x d s
\end{aligned}
$$

for any $L>0$. Therefore, $v^{\delta}(\cdot, t)$ is Cauchy in $L_{l o c}^{2}(\mathbb{R})$ for any $t \in \mathcal{D}$.

We conclude the proof of Theorem 1.1 by observing that compactness properties (4.5)-(4.8) will guarantee that $(v, u, e)$ is a weak solution of (1.1)-(1.3) satisfying regularity estimates (1.6)-(1.8). The proof of the lower bound for $e$ in (1.6) is standard and can be found in Wong [6].

\section{REFERENCES}

[1] G.-Q. Chen, D. Hoff, K. Trivisa, Global Solutions of the Compressible Navier-Stokes Equations with Large Discontinuous Initial Data, Acta Math. Appl. Sin. Engl. Ser., 18 (2002), pp. $15-36$.

[2] D. Hoff And E. Tsyganov, Uniqueness and continuous dependence of weak solutions in compressible magnetohydrodynamics, ZAMP, 56 (2005), pp. 791-804.

[3] D. Hoff And E. Tsyganov, Time analyticity and Backward Uniqueness of Weak Solutions of the Navier-Stokes Equations for Multidimensional Compressible Flow, submitted.

[4] S. JiAng AND A.A. ZlotniK, Global well-posedness of the Cauchy problem for the equations of a $1 D$ viscous heat-conducting gas with Lebesgue initial data, Proc. Roy. Soc. Edinburgh, 134A (2004), pp. 239-260.

[5] E. Tsyganov, On time analyticity of weak solutions of the compressible Navier-Stokes equations, Physica D, 227 (2007), pp. 97-103.

[6] D. WANG, On the global solution and interface behaviour of viscous compressible real flow with free boundaries, Nonlinearity, 16 (2003), pp. 719-733.

[7] A.A. Zlotnik And A.A. Amosov, Stability of generalized solutions to equations of onedimentional motion of viscous heat conducting gases, Math. Notes, 63 (1998), pp. 736-746. 
E. TSYGANOV 